\title{
SPIN DIRECTIONS IN EXCHANGE COUPLED RE-TM DOUBLE LAYER FILMS WITH IN-PLANE MAGNETIC LAYER
}

\author{
R. MALMHÄLL*, T. NIIHARA, H. MIYAMOTO and M. OJIMA \\ Central Research Laboratory, Hitachi Ltd., Kokubunji, Tokyo 185, Japan \\ *On leave from Institute of Optical Research, S-100 44 Stockholm, Sweden
}

\section{Introduction}

Exchange coupling between amorphous rare earth-transition metal (RE-TM) layers is of large interest due to the possibility for direct overwrite in magneto-optical recording [1]. It is therefore very important to be able to model the spin orientations in the different layers and the influence of exchange interaction. In this report, Hall effect has been employed for studying exchange coupling between TbDyFeCo write layer (W) and TbFeCo memory layer (M) with various interface (I) layers, using the fact that Hall effect in magnetic materials is strongly dependent on the magnetic state of the material due to the existence of the extra-ordinary Hall effect.

\section{Experimental details}

Several samples of RE-TM alloy films were made by if sputtering. The samples studied here had, for example, an interface layer of in-plane magnetic anisotropy GdCo alloy (I-layer) as shown in Fig.1. The magnetic properties of $\mathrm{W}$-layer and $\mathrm{M}$-layers were chosen so that $\mathrm{W}$-layer was magnetically RE-dominated and M-layer TM-dominated at room temperature. Typical dimensions of the Hall samples were $3 \times 10 \mathrm{~mm}^{2}$ and electrical leads of thin gold wires were indium-soldered to the films. The Hall voltage was measured with an alternating current and a dc magnetic field technique using a lock-in amplifier. The maximum field used was $17 \mathrm{kOe}$.

\section{Results and Discussion}

The Hall hysteresis loops for each alloy measured on single layer films are indicated in Fig. 1 , where the loop polarities are consistent with the dominating sublattice [2]. Figure 2 gives the Hall loops for the tri-layer film, one for each of the two possible directions of the M-layer magnetization. It can be seen that if $M$-layer was switched in a positive field $(M+)$, the loop is shifted to the right, and in the opposite direction when it was switched with a negative field (M-). However, it was also found that the Hall curve exhibited a high field slope for exchange coupled films with I-layer. The slope after switching of W-layer can be seen more clearly in Fig.3. The four curve sections corresponds to (1) W+ M-, (2) W+ M+, (3) W- M+ and (4) W- M-. The curves (2) and (4) were found to approach the field axis with increasing temperature and to cross over into the 3rd quadrant at about $50 \mathrm{C}$. The slope of curve (1) and (3) was seen to increase with higher temperature.

From the observed Hall loops, the spin orientations of the different layers were deduced and are illustrated in Fig.4. Thus, the GdCo I-layer appears to be almost fully magnetized in the normal direction at zero field, and an in-plane wall exists inside the W-layer at zero field when the $M$ and $W$ layer net magnetizations are parallel. The Hall results also indicate that the in-plane wall moves to the interface between the $\mathrm{W}$ and I-layer at $\mathrm{T} \approx 50 \mathrm{C}$, and further into the I-layer at higher $\mathrm{T}$.

\section{Conclusions}

It has been demonstrated how Hall effect measurement can be used to give insight into the effect of exchange interaction on the magnetization directions in multilayer films. In the case of an inplane magnetic anisotropy TM-dominated GdCo interface layer at room temperature, the GdCo layer will be almost fully magnetized in the normal direction at zero applied magnetic field, and an in-plane domain wall will be present inside the TbDyFeCo layer at zero field when the net magnetizations of 
the two RE-TM layer are parallel. It was also found that the in-plane wall shifts to the interface between the TbDyFeCo and GdCo layers at a temperature of about $50 \mathrm{C}$ and continues into the interface layer at higher temperatures.

\section{References}

[1] J. Saito, et al., Jap.J.Appl.Phys., 26, Suppl. 26-4, 155 (1987).

[2] R. Malmhäll, J.Appl.Phys., 54, 5128 (1983).

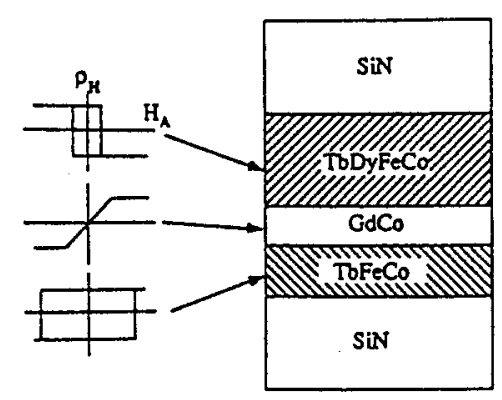

Fig.1. Structure of exchange coupled RE-TM film and Hall resistivity attributed to each layer.

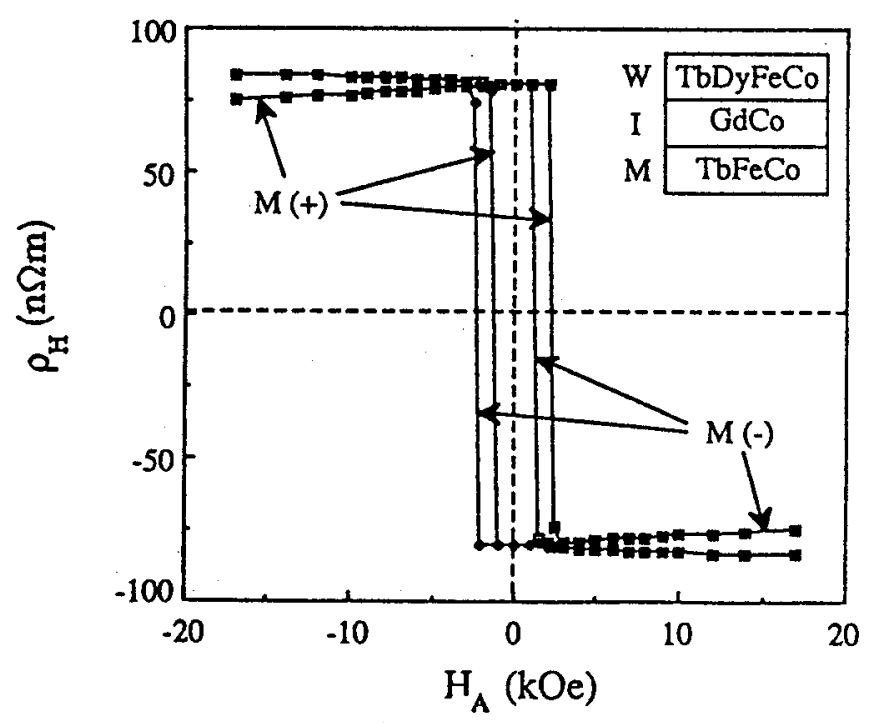

Fig.2. Hall resistivity vs applied magnetic field for $\mathrm{TbDyFeCo} / \mathrm{GdCo} / \mathrm{TbFeCo}$ film at room temperature where the TbFeCo layer was switched previously in a positive field $(M+)$ and a negative field $(M-)$.

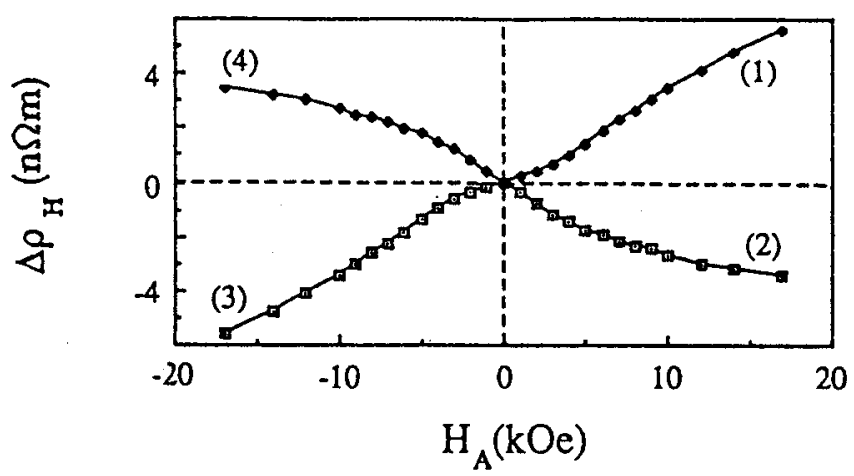

Fig.3. Hall resistivity change as a function of applied magnetic field, corresponding to an enlargement of the data in Fig. 2.

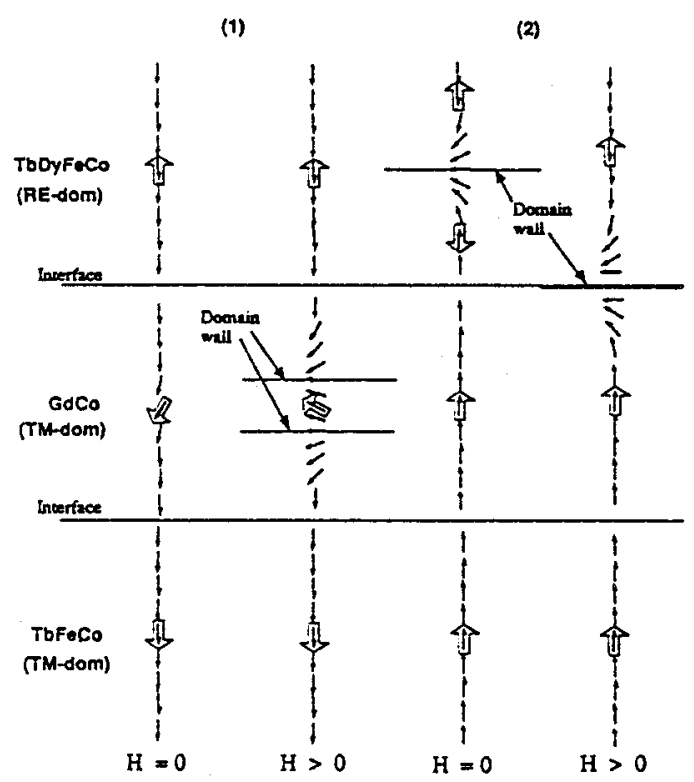

Fig.4. Spin orientations in exchange coupled $\mathrm{TbDyFeCo} / \mathrm{GdCo} / \mathrm{TbFeCo}$ film at room temperature. 